\title{
Spinal cord injury: treatments and medications
}

\section{Introduction}

Spinal Cord Injury (SCI) is currently one of the leading causes of disability in the United States. According to the National Institute of Neurological Disorders and Stroke (NINDS), accidents, domestic violence, falls, account for about 10,000 cases of spinal cord injuries each year. The demographics reported by the National Spinal Cord Injury Association (NSCIA), causes range from motor vehicle accidents $(44 \%)$, domestic violence $(24 \%)$, falls $(22 \%)$, physical activities, sports and others $(10 \%)$.

The extent of the injury depends on the area of the initial injury primarily, but also the post-accident medical attention is highly important. The time interval between the injury and the treatment is critical and can determine the extent of severity, complication and recovery.

Injuries to the higher regions of the spinal cord (cervical region) can lead to more severe injuries leading to complete paralysis of arms, hands, trunk, and legs (also termed as tetraplegia or quadriplegia), requiring 24hours assistance, and causing urinary and sexual dysfunction. Injuries to the lower parts (thoracic and lumbar region) mostly lead to paralysis of the trunk and the legs (also termed as paraplegia), partial aid requirement, and restricted movement on the wheelchair. Spinal Cord injury can be complete or incomplete. In a complete injury there is no sensation with total disruption of signals below the injury. In cases of incomplete injury, there is partial sensation and transduction of signals below the level of injury leading to partial paralysis.

A case of SCI comes with several phases of challenge. The initial phase in the injury, which involves physical damage to the spinal cord, severing a part of it, causing considerable damage to the axons, neighboring cells, intolerable pain, Neurotrauma, emotional shock, broken or fractured vertebrae just to list a few of them. After the initial impact if possible the patient should not be moved without expert assistance from emergency professionals who are specially trained for these situations. It is very important that the head, neck and shoulder be immobilized to avoid further injury. The patient is usually transferred to Intensive Care Unit and treated accordingly. The second phase of the
Volume 2 Issue 4 - 2016

\section{Sreyashi Samaddar}

Department of Anatomy, City University of New York, USA

Correspondence: Sreyashi Samaddar, Department of Anatomy, City University of New York, USA,

Email sreyashi.samaddar@gmail.com

Received: May 09, 2016 | Published: May 23, 2016

injury is called the secondary injury and this phase is a progressively degenerating phase in most of the cases. Here the damage starts at a molecular level with a cascade of events that hinders the recovery process. The molecular events occurring include, inflammation leading to migration of immune cells to the area of the injury, killing the surviving neurons and oligodendrocytes that could have aided in the recovery process. Inflammation results in the formation of free radicals, which alter the chemical structure of cells. Excitotoxicity ensues after SCI, due to the excessive release of the neurotransmitter, glutamate. Excessive release of calcium also results in this excessive glutamate release, which in turn activates several signaling cascades simultaneously. Apoptosis is one other very common phenomenon that starts kicking in a few days post-injury. Nerve cells that could have been beneficial in the process of regeneration undergo apoptosis and attenuate the recovery process. Mitochondrial damage is one of the most significant secondary effects of SCI. Vascular damage and hypoxia causes mitochondrial dysfunction that would otherwise serve as an energy buffer during pathological conditions. Excessive release of calcium also damages mitochondria and depletes ATP leading to cell death. Astrocytes accumulate forming glial scar, obstructing axon growth, blocks communication of growth factors, blood flow, oxygen and nutrients to the injury site. All these biochemical, molecular and vascular events work in a cascade and ultimately slow down the recovery process (Table 1$){ }^{1}$

Table I Summary of events of secondary neuro-injury following SCI

Vascular events

Biochemical events breakdown of blood-spinal cord barrier oedema formation ischemia and hypoxia release of vaso active substances excitotoxicity formation of free radicals and nitric oxide release of proteases damage of mitochondrias energy depletion

Cellular events

invasion of neutrophils activation of resident microglia invasion of peripheral macrophages infiltration of lymphocytes activation of astrocytes apoptosis of oligodendrocytes Wallerian degeneration

Table adapted from Hausmann ON (n.d.). Post-traumatic inflammation following spinal cord 


\section{Treatment and therapies}

In this age of scientific marvels and discoveries, unfortunately there is still no complete cure for SCI. Majority of the treatments and therapies involved in SCI, are basically aimed at preventing further damage and helping patients restore normal life activities as much as possible by external assistance. There is still date no way to reverse the damage caused by the initial accident. The different phases of treatment include

\section{Medication}

Methylprednisolone (IV) if administered within the first eight hours of injury in known to bring about mild improvement for some patients. It is not a cure, but acts as an anti-inflammatory agent. Medications also include pain control drugs, drugs to control muscle spasticity, bladder and bowel functioning and even anti-depressants.

\section{Surgery}

Surgery is sometimes necessary to remove and re-align broken or fractured vertebrae, prevent pain or deformities due to compression on the spine.

\section{Rehabilitation}

A crucial part of the treatment after the initial medical intervention is rehabilitation. Rehabilitation involves a team including physical therapist, occupational therapist, rehabilitation nurse, social worker, and dietician, recreation therapist who work to help in strengthening muscle, regaining motor skills and restoring normal activities as much as possible. They also work with the patient and the patient family to provide emotional support and in management of the trauma.

\section{Modern technologies}

Medical devices are common to help restore independence and mobility. Modern automated electric wheelchairs capable to tasks like climbing stairs, travel purposes, elevate a seated passenger are commonly used. Computers are used as powerful tools for patients with limited hand function. Electronic aids for daily activities that can be turned on and off, voice-controlled and even remote controlled. Electrical stimulation systems are devices that use electrical stimulation to control hand and leg movement helping patients to stand, walk, and other actions.

\section{Medications currently used}

Medication currently available for treating SCI, does not reverse the damage caused, but is targeted towards prevention of further damage due to secondary injuries.

\section{Methylprednisolone (MP)}

A corticosteroid or glucocorticoid drug is used for its antiinflammatory effects. The National Acute Spinal Cord Studies (NASCIS), has evidence of considerable improvement in motor function in patients with complete and incomplete spinal cord injuries with the administration of high doses of MP within eight hours of the initial injury. NASCIS trials have led the administration of MP a standard procedure in the treatment of SCI patients. MP is also used to treat multiple sclerosis and vestibular neuritis (Figure 1).

\section{Mechanism of action}

MP is known to exert its effect in many different ways. It suppresses the gene expression of pro-inflammatory cytokines, affects overall decrease in the general population of CD4+ T cells, inhibits IL-4 signaling pathway by down -regulation of IL-4-induced IL-4 receptor expression. MP inhibits T-cell activation, enhances antigen uptake of dendritic cells, hinders dendritic cell maturation, and decreased production of TNF-alpha, IL-6, IL-12.

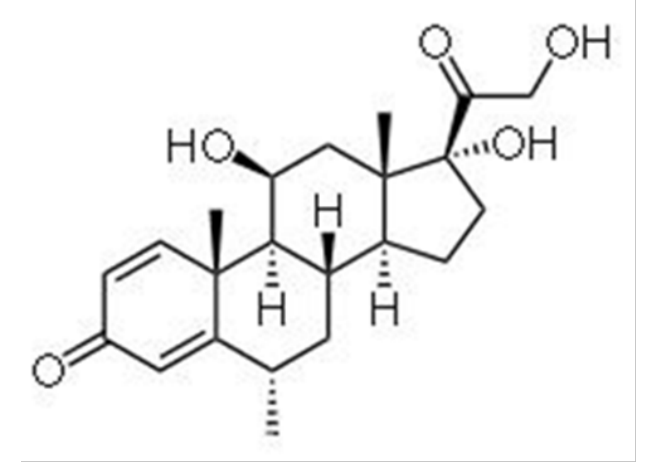

Figure I Chemical structure of methylprednisolone.

One of the major concerns involving secondary injury is cytotoxicity from the free radicals. One such molecule is nitric oxide (NO). NO and TNF-alpha causes myelin damage and oligodendrocyte destruction. MP causes reduced production of TNF-alpha and could in turn affect reduced production of NO. High-dose of MP is also known to inhibit Lipid Peroxidation, which in turn leads to preservation of $\mathrm{Ca}+, \mathrm{Na}+\mathrm{K}+$ homeostasis, inhibition of cytoskeletal damage, attenuation of glutamate release, restoration of aerobic metabolism, and preservation of Spinal cord blood flow. Thus MP interferes with mediators of inflammatory response and suppresses immune response. ${ }^{2}$ Long-term usage of glucocorticoids, has several side effects like hyperglycemia, increased vulnerability to infection, weight gain, osteoporosis, psychosis, edema, hypertension, glaucoma to name a few. The down side of the glucocorticoid-related side effects led to the discovery of a non-gluco corticoid, '21-aminosteroids' or 'lazaroids'.

\section{Tirilazad mesylate}

21-aminosteroids (lazaroids) are inhibitors of lipid membrane peroxidation and oxygen free radical scavengers. NASCIS III clinical trials demonstrated that the non-glucocorticoid Tirilazad is as effective as 24 hour MP therapy. Moreover, with minimal side effects, non-glucocorticoid, proved to be a safer and effective alternative in treating SCI (Figure 2).

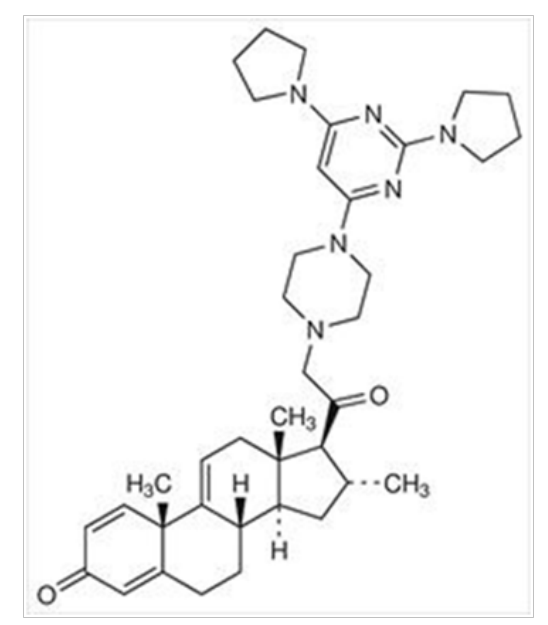

Figure 2 Chemical structure of Tirilazad. 


\section{Riluzole}

Riluzole is a sodium channel-blocking benzothiazole anticonvulsant currently used in the treatment of Amylotrophic Lateral Sclerosis (ALS) (Figure 3).

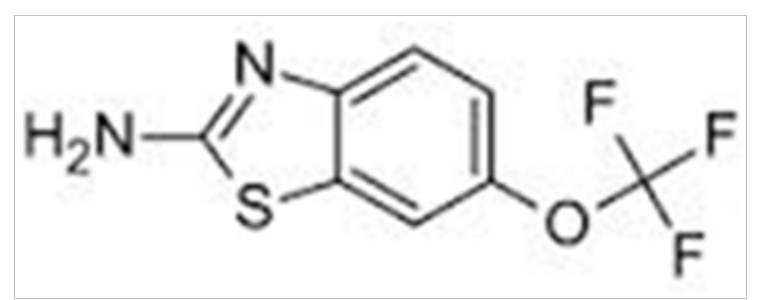

Figure 3 Chemical structure of Riluzole.

\section{Mechanism of action}

An important secondary injury cascade is the development of an ionic imbalance particularly of sodium and calcium. The injuryinduced trauma leads to the activation of voltage-sensitive sodium channels, resulting in increased intracellular sodium concentration. Increased sodium concentration leads to increased intracellular calcium concentration and increased neuronal release of excitatory release of neurotransmitter glutamate. Excessive release of glutamate caused excite-toxicity and local cell death. Riluzole compared to other novel sodium channel blockers like Phynetoin, demonstrated significantly larger degree of functional recovery than other treatment groups. Riluzole's neuroprotective role is due to its ability to prevent both sodium and calcium influx and at the same time glutamate release. It is an approved drug for ALS, and clinical trials are underway for SCI. ${ }^{3}$

\section{Conclusion}

The scientific community has been working relentlessly to find a potential therapy or a drug that can make the process of degeneration slower, and ameliorate the process of recovery. Unfortunately the nature of the injury with multiple factors interacting with each other makes it even more challenging to address the problem with one single target. Apart from attenuating the inflammation, excitotoxicity, cell death, studies are underway for axon regeneration, new cell formation, which could rebuild the lost neural circuit.The other important factor that adds up to the recovery process is the emotional balance of the affected individuals. The shock and the trauma, is beyond explanation and the fact that SCI leads to dependence and requirement for assistance to some extent, creates a toll on the patients and their families. Recovery is a wholesome process, biochemical, molecular, motor, sexual, and also psychological. With the discovery of target molecules and innovative devices hopefully treating SCI will be easier in the years to come.

\section{Acknowledgements}

None.

\section{Conflict of interest}

Author declares that there is no conflict of interest.

\section{References}

1. Hausmann ON Post -traumatic inflammation following spinal cord injury. Spinal Cord. 41(7):369-378.

2. Sloka JS, Stefanelli M. The mechanism of action of methylprednisolone in the treatment of multiple sclerosis. Multiple Sclerosis. 2005;11(4):425-432.

3. Fehlings MG, Wilson JR, Frankowski RF, et al. Riluzole for the treatment of acute traumatic spinal cord injury: rationale for and design of the NACTN Phase I clinical trial. J Neurosurg Spine. 2012;17(Suppl1):151-156. 